\title{
Multiple Primary Choledocholithiasis in Sickle Cell Disease
}

\author{
Perla Vicari, Marco Vinicio Fanucchi Gil, Rita de Cássia Rosário Cavalheiro \\ and Maria Stella Figueiredo
}

\begin{abstract}
Choledocholithiasis can be caused by either primary or secondary bile duct stones. Although patients with sickle cell disease (SCD) are at high risk of development of pigmented gallstones due to chronic hemolysis, primary choledocholithiasis in SCD is very uncommon. The delay in the diagnosis of biliary tract pathology can occur in patients who had a prior cholecystectomy. There is a possible relationship between prior cholecystectomy and the subsequent occurrence of primary common bile duct stones, due to the higher probability of infection of the biliary system. Here, we report an unusual and severe case of multiple primary choledocholithiasis, associated with pancreatitis, in a patient with SCD, fourteen years after cholecystectomy.
\end{abstract}

Key words: sickle cell disease, choledocholithiasis, gallstones

(Inter Med 47: 2169-2170, 2008)

(DOI: 10.2169/internalmedicine.47.1326)

\section{Introduction}

Choledocholithiasis can be caused by either primary or secondary bile duct stones. The primary duct stones originate in the bile duct and the secondary stones have exited the gallbladder. Approximately $95 \%$ of this complication is due to the migration of gallstones. Therefore, if the gallbladder is still in place, or only recently removed, the origin of the stone in the common duct is impossible to prove (1).

Although patients with sickle cell disease (SCD) are at high risk of development of pigmented gallstones due to chronic hemolysis, primary choledocholithiasis is very uncommon (2). We report an unusual case of multiple primary choledocholithiasis, associated with pancreatitis, in a patient with SCD, fourteen years after cholecystectomy.

\section{Case Report}

A 27-year-old homozygous sickle cell man was admitted for upper abdominal pain, choluria, increase of jaundice, nausea and vomiting. Fourteen years previously cholecystectomy was performed due to symptomatic cholelithiasis. During this time, the patient was asymptomatic and without residual stones on abdominal ultrasound.

On admission, blood hemoglobin was $7.6 \mathrm{~g} / \mathrm{dL}$, platelet
$483 \times 10^{3} / \mathrm{mm}^{3}, 12 \times 10^{3} / \mathrm{mm}^{3}$, total serum bilirubin $32.5 \mathrm{mg} /$ $\mathrm{dL}$, conjugated serum bilirubin $17.5 \mathrm{mg} / \mathrm{dL}$, serum alanine aminotransferase level $43 \mathrm{U} / \mathrm{L}$, serum aspartate aminotransferase $74 \mathrm{U} / \mathrm{L}$, gamma glutamyl transferase $340 \mathrm{U} / \mathrm{L}$ and amylase $640 \mathrm{U} / \mathrm{L}$. Abdominal ultrasound showed hepatomegaly and choledocholithiasis with intrahepatic and choledocho ducts dilation. Cholangio magnetic resonance confirmed dilation of the intra and extrahepatic ducts with choledocholithiasis (Fig. 1). Laparotomy was performed. Operative cholangiography showed numerous calculi in choledocho duct. Choledocholithotomy of multiple pigment stones combined with T-tube drainage was performed.

One day after surgery, the patient developed left bronchopneumonia, which was treated with cefepime. The subsequent course was marked by acute chest syndrome and sepsis. Multiorgan failure and death occurred fourteen days after laparotomy.

\section{Discussion}

Gallstones are a frequent complication in SCD. The development of pigment gallstones due to chronic hemolysis is age dependent: $15 \%$ under 10 years, $22 \%$ between 10 and 14 years and $36 \%$ between 15 and 18 years, and reported prevalence of $50 \%$ by the age of 22 years $(3,4)$.

The diagnosis of primary common duct stones depends on 


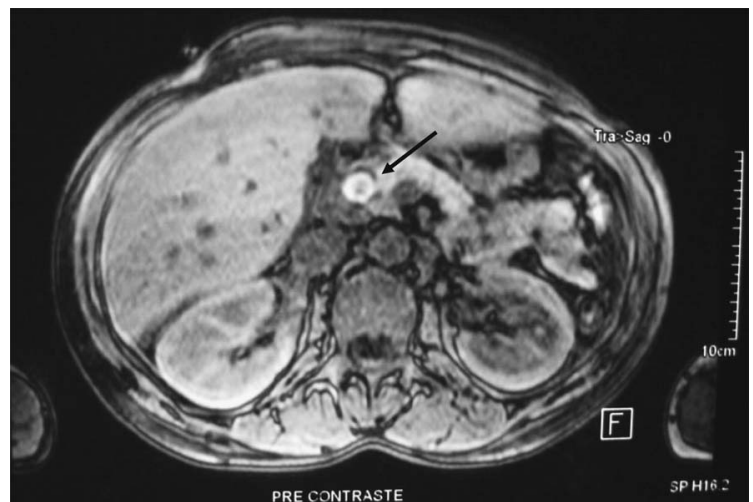

Figure 1. Cholangio magnetic resonance with intra and extrahepatic ducts dilation with choledocholithiasis (arrow).

the following criteria: (1) previous cholecystectomy with or without common duct exploration; (2) at least a two-year asymptomatic period following the initial biliary tract surgery; and (3) no evidence of biliary stricture resulting from prior surgery $(1,5)$. Choledocholithiasis has been noted in around $18 \%$ of patients with SCD undergoing cholecystectomy (2). However, the prevalence of primary choledocholithiasis in SCD has not been established. In the general population, only $5 \%$ of choledocholithiasis are primary bile duct stones (1).

Symptomatic biliary tract disease is difficult to diagnosis in patients with SCD, who frequently can have acute abdominal pain, fever and jaundice, as symptoms of vaso- occlusive crisis. Equally, distinguishing acute cholecystitis from sickle hepatic crisis may be difficult because of their similar clinical presentations, mainly in patients with cholecystectomy history $(2,4,6)$.

Perhaps patients who have had a prior cholecystectomy have a higher probability of infection of the biliary system, and this may be one of causes of occurrence of primary common bile duct stones after cholecystectomy (7). The present patient had undergone cholecystectomy fourteen years previously and choledocholithiasis had never been seen during his follow-up. According to the current views, associations of bile stasis and infection could explain intrahepatic stones formation (6). However, there was no evidence of these in our patient.

\section{Conclusion}

In summary, this case is a rare case of multiple primary choledocholithiasis in SCD, with a diagnosis complicated by the previous history of cholecystectomy. The delay in the diagnosis of biliary tract pathology can occur when the patient presents a long symptom-free interval since cholecystectomy. In recent years, several minimally invasive treatments for choledocholithiasis are becoming popular and reducing complications (8). Although uncommon, early recognition of this entity is essential to avoid life-threatening complications.

\section{References}

1. Saharia PC, Zuidema GD, Cameron JL. Primary common duct stones. Ann Surg 185: 598-604, 1977.

2. Banerjee S, Owen C, Chopra S. Sickle cell hepatopathy. Hepatology 33: 1021-1028, 2001.

3. Suell MN, Horton TM, Dishop MK, Mahoney DH, Olutoye OO, Mueller BU. Outcomes for children with gallbladder abnormalities and sickle cell disease. J Pediatr 145: 617-621, 2004.

4. Related Articles, LinksCurrò G, Meo A, Ippolito D, Pusiol A, Cucinotta E. Asymptomatic cholelithiasis in children with sickle cell disease: early or delayed cholecystectomy? Ann Surg 245: 126-129, 2007.

5. Wu SD, Tian Y, Kong J, Ding RY, Jin JZ, Guo RX. Possible relationship between cholecystectomy and subsequent occurrence of primary common bile duct stones: a retrospective review of data. Hepatobiliary Pancreat Dis Int 6: 627-630, 2007.

6. Hillaire S, Gardin C, Attar A, et al. Cholangiopathy and intrahepatic stones in sickle cell disease: coincidence or ischemic cholangiopathy? Am J Gastroenterol 95: 300-301, 2000.

7. Wu SD, Tian Y, Kong J, Ding RY, Jin JZ, Guo RX. Possible relationship between cholecystectomy and subsequent occurrence of primary common bile duct stones: a retrospective review of data. Hepatobiliary Pancreat Dis Int 6: 627-630, 2007.

8. Uchiyama K, Onishi H, Tani M, et al. Long-term prognosis after treatment of patients with choledocholithiasis. Ann Surg 238: 97102, 2003.

(C) 2008 The Japanese Society of Internal Medicine http://www.naika.or.jp/imindex.html 